\title{
A desktop extreme ultraviolet microscope based on a compact laser-plasma light source
}

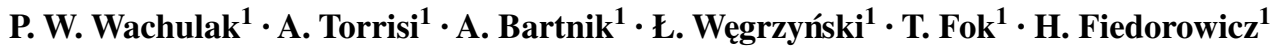

Received: 6 September 2016 / Accepted: 14 November 2016 / Published online: 24 December 2016

(c) The Author(s) 2016. This article is published with open access at Springerlink.com

\begin{abstract}
A compact, desktop size microscope, based on laser-plasma source and equipped with reflective condenser and diffractive Fresnel zone plate objective, operating in the extreme ultraviolet (EUV) region at the wavelength of $13.8 \mathrm{~nm}$, was developed. The microscope is capable of capturing magnified images of objects with 95-nm full-pitch spatial resolution ( $48 \mathrm{~nm} 25-75 \% \mathrm{KE}$ ) and exposure time as low as a few seconds, combining reasonable acquisition conditions with stand-alone desktop footprint. Such EUV microscope can be regarded as a complementary imaging tool to already existing, well-established ones. Details about the microscope, characterization, resolution estimation and real sample images are presented and discussed.
\end{abstract}

\section{Introduction}

Recent developments in nanoscience and nanotechnology require nanoscale imaging tools. For that, electromagnetic radiation in the extreme ultraviolet (EUV) spectral range ( $\lambda=10-121 \mathrm{~nm}$ wavelength [1]) allows shifting the diffraction limit into a nanometer range [2, 3]. Much work has already been done developing different photon-based imaging techniques and schemes, according to the Rayleigh criterion, which states that the light of shorter wavelength improves the diffraction-limited spatial resolution. Some examples of this demonstrate the use of synchrotronbased sources [4] reaching spatial resolution of $\sim 10 \mathrm{~nm}$ [5] or using $13.5 \mathrm{~nm}$ wavelength for lithography-related

P. W. Wachulak

wachulak@gmail.com

1 Institute of Optoelectronics, Military University of Technology, Kaliskiego 2 Str., 00-908 Warsaw, Poland research, such as mask inspection [6, 7]-reaching 22-nm half pitch resolution or lithography [8], as well as free electron lasers [9] for coherent diffraction imaging (CDI) schemes [10]. These facilities, although state of the art and dedicated to cutting-edge science experiments, are not "user friendly," with limited user access and require high maintenance costs, because of their scale and complexity. Another approach is to use tabletop high-order harmonic (HHG) sources [11] for sub-100-nm spatial resolution imaging [12]; however, typical $10^{-6}-10^{-5}$ HHG conversion efficiency is very low and often does not allow for a proper reconstruction [13], the system is very complicated, and typical CDI requires time-consuming numerical data processing. Ptychographic schemes, although providing very high spatial resolution, are serial in nature, extensively time-consuming and computationally demanding.

To partially overcome these limitations, other compact EUV sources, such as discharge [14], Z-pinch [15] or laserproduced plasma sources [16], coupled to zone plates or Schwarzschild mirrors, were used. The first one is compact and shows very good spatial resolution, but requires often ( $\sim 30 \mathrm{k}$ pulses) capillary replacements, the second one demonstrates quite low performance in terms of spatial resolution and field of view exploiting inadequate mode of imaging for lithographic mask inspection, while the last one requires debris mitigation schemes.

The use of compact, short-wavelength sources often does not allow for high signal-to-noise ratio image acquisition. An example of that are recent developments in soft X-ray (SXR) microscopy in so-called water window, such as a compact soft X-ray microscope based on a single nitrogen gas jet, capable of resolving features $\sim 100 \mathrm{~nm}$ later improved to $\sim 50 \mathrm{~nm}$ in size providing high spatial resolution; however, the exposure time for Siemens star test pattern was equal to $1-2 \mathrm{~h}$, limiting the usability of 

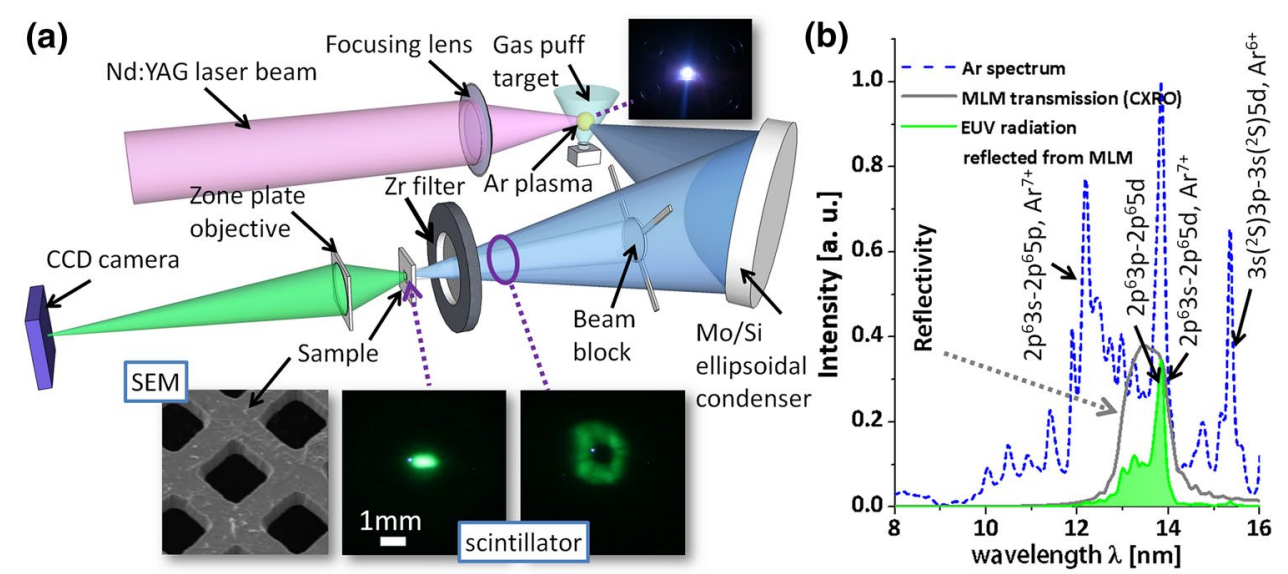

Fig. 1 Scheme of the EUV microscope. Small insets show plasma image in the visible light wavelength range (top), SEM image of the test object $(\mathrm{Cu}$ mesh) and scintillator images of intensity distribution of the EUV radiation in (middle-bottom inset) and out (right-bottom

such system to a few images per day [17, 18]. Much more rapid exposures of $60 \mathrm{~s}$ were required to image objects with 40-nm spatial resolution, employing a high average power laser system for plasma generation, occupying, however, several optical tables [19], which in turn limit future possibility of commercialization. Generally speaking, a tradeoff in the short-wavelength EUV and SXR imaging can be seen between the performance, complexity and compactness of the system, which is still a major obstacle in widespread short-wavelength photon-based microscopes.

Thus, in this work, we try to partially overcome presented limitations, demonstrating a simple, very compact full-field EUV microscope, which is capable of resolving sub-100-nm features, requires short exposure time and has a desktop footprint. The use of a laser-plasma EUV source based on a gas-puff target [20, 21] eliminates debris production problem of solid targets. The source, which was already successfully employed in the SXR microscopy [22], is simple in construction and was sufficiently bright to be a driver for the EUV microscope that is user friendly and can be operated just by one person. The microscope requires no sample preparation and offers high reproducibility of images and preservation of the sample integrity. Moreover, it is already well known that the water-window radiation is dedicated for imaging of biological samples, due to a natural contrast between carbon and water constituents of the living cells. It is true for imaging internals of the cells. Herein, we propose that the EUV microscopy can be used for imaging biological samples as well; it is more adequate, however, for imaging thin external cell features, such as morphology of the cellular membranes or other external features, such as flagella, which produce high contrast in the EUV range, while it may be overlooked in the water window. inset) of the focal plane of the condenser mirror. Spectrum of the Ar plasma emission (b) (blue-dashed line). The condenser reflectivity is depicted in solid gray line. The green line spectrum shows radiation reflected from the condenser, used for subsequent imaging

\section{EUV microscope construction}

A scheme of the EUV microscope is shown in Fig. 1a. An Nd:YAG laser pulse (NL302, Eksma), $\lambda=1064 \mathrm{~nm}$, $500 \mathrm{~mJ} / 4 \mathrm{~ns}$, is focused using a lens onto a double-stream $\mathrm{Ar} / \mathrm{He}$ gas-puff target $[23,24]$, produced by an electromagnetic double-nozzle valve [25] resulting in formation of a plasma. The optimum Ar/He pressure for efficient EUV emission from such plasma was found to be 10 and 6 bar, respectively. In order to collect the radiation emitted from the Ar plasma, Fig. 1b-dashed line, and to spectrally narrow the emission, an ellipsoidal off-axis mirror with $\mathrm{Mo} /$ Si multilayer coating (MLM), acting as a condenser, was used.

The condenser (Reflex, Prague, Czech Republic and IOF, Jena, Germany) reflects radiation at the wavelength of $13.5 \pm 0.5 \mathrm{~nm}$ at $45^{\circ}$ incidence angle, Fig. 1b-gray solid line [26]. Geometry of the condenser and its filtering property significantly reduces the overall footprint of the entire system, as shown in photograph depicted in Fig. 2, and improves the monochromatic photon flux at the sample plane. The condenser position was optimized for maximum energy and uniformity of the sample illumination. The scintillator EUV images in focal plane (left) and out of plane (right), obtained with P43 scintillator screen (Proxitronic), are depicted in insets in Fig. 1a. The FWHM size of the spot illuminating the sample was $1.5 \times 0.8 \mathrm{~mm}^{2}$. To suppress axial EUV radiation, a circular beam stop $\sim 3 \mathrm{~mm}$ in diameter was placed $5 \mathrm{~cm}$ upstream the sample. To eliminate longer wavelengths emitted from $\operatorname{Ar}$ plasma $(\lambda>16 \mathrm{~nm})$, a 100-nm-thick free-standing zirconium filter (Lebow) was used. The object-copper TEM mesh (SPI Supplies), SEM image shown in small inset in Fig. 1a-was located in the second focal plane of the condenser, at a distance 
of $254 \mathrm{~mm}$ from the condenser. The object was imaged in transmission mode, using a Fresnel zone plate objective FZP (Zone Plates Ltd., UK) (diameter $200 \mu \mathrm{m}$, number of zones 1000 and the outer zone width $\Delta r=50 \mathrm{~nm}$ ) onto a CCD camera, iKon-M, DO-934-BN (Andor), 1 Mpixel in size. The FZP was fabricated using e-beam lithography in a 200-nm-thick PMMA layer, deposited on top of a 50-nmthick $\mathrm{Si}_{3} \mathrm{~N}_{4}$ membrane, which resulted in first-order diffraction efficiency of $\sim 20 \%$. The theoretical spatial resolution (Rayleigh or full-period resolution) is $1.22 \Delta r=61 \mathrm{~nm}$, and a depth of focus (DOF) is equal to $\sim 740 \mathrm{~nm}$.

The numerical aperture of the condenser $\left(<\mathrm{NA}_{\mathrm{CH}}\right.$, $\mathrm{NA}_{\mathrm{CV}}>=<0.11,0.15>$, in horizontal and vertical directions) matches the NA of the FZP objective $\left(\mathrm{NA}_{\mathrm{o}}=0.137\right)$, providing spatially incoherent object illumination [27]. The geometrical magnification of the objective was $410 \times$; however, it can be changed by adjusting the FZP-CCD distance up to $\sim 700 \times$, at which the signal-to-noise ratio in the images becomes low. For accurate positioning of the FZP along the optical axis, a closed-loop piezo-stage (LPS45, PI miCos GmbH, Germany) was used (positioning

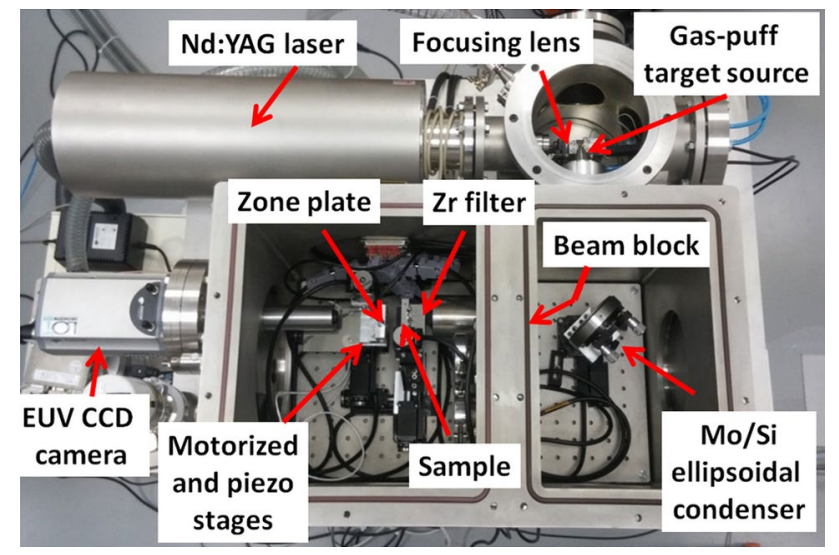

Fig. 2 Photograph of the microscopy system showing construction of the compact EUV microscope repeatability of $50 \mathrm{~nm})$. The entire microscope has a very compact size: $(\mathrm{W} \times \mathrm{D} \times \mathrm{H})(100 \times 70 \times 160) \mathrm{cm}^{3}$. Such EUV microscope, to our knowledge, is currently one of the most compact EUV imaging tools reported in the literature. A photograph of the internals of the EUV microscope is depicted in Fig. 2.

\section{EUV microscope characterization and resolution estimation}

The photon flux in 13-14 nm spectral range-green curve in Fig. 1b-for a single EUV pulse was $(1.4 \pm 0.1) \cdot 10^{11}$ photons, based on 23 measurements, in a focus of the condenser, measured with a $10 \times 10 \mathrm{~mm}^{2}$ AXUV100 detector (IRD Inc., USA), coated with a thin layer of $\mathrm{Zr} / \mathrm{C}$ $(200 / 50 \mathrm{~nm})$. This results in $\sim 1.7 \cdot 10^{8}$ photons/pulse in the field of view (FOV) of the microscope. For spectral measurements, a transmission grating spectrometer with 200-nm pitch $\mathrm{Si}_{3} \mathrm{~N}_{4}$ grating was used. Figure $1 \mathrm{~b}$ depicts Ar spectrum and spectrum reflected from the condenser mirror, in a $\Delta \lambda=1-\mathrm{nm}$ EUV band, used for subsequent imaging. In this band, the predominant spectral line is at $\lambda=13.84 \mathrm{~nm}-2 p^{6} 3 s-2 p^{6} 5 d$ transition in $\mathrm{Ar}^{7+}$ [28].

For this demonstration, a typical EUV image of the test object-Cu mesh-is shown in Fig. 3b. The image of the object was acquired by integrating 200 EUV pulses over 20-s exposure time. During image acquisition, the backilluminated $\mathrm{CCD}$ camera was cooled down to $-20^{\circ} \mathrm{C}$ and the readout rate was set to $50 \mathrm{kHz}$ to minimize the noise. The features of the mesh are clearly visible. The FOV of the EUV image was $\sim 34 \times 34 \mu \mathrm{m}^{2}$.

To determine the spatial resolution of the microscope, a well-established "knife-edge" (KE) test [2] was employed. The KE test was performed across $2 \mu$ range, located between two dotted lines in Fig. 3b. This distance corresponds to $\sim 61$ pixels (each is $33 \times 33 \mathrm{~nm}^{2}$ in size) in the EUV image. Typical KE measurement, depicted in Fig. 3a, shows the KE 10-90\% intensity change over a distance of
Fig. 3 A 10-90\% knife-edge spatial resolution test (Rayleigh full pitch) and 25-75\% KE (a) for the EUV image (b). The resolution was estimated from the intensity profile obtained from the EUV image (b). Encircled feature in (b) is a mesh defect $100 \times 300 \mathrm{~nm}^{2}$ in size. The image (b) was acquired by integrating $200 \mathrm{EUV}$ pulses over 20 s exposure time
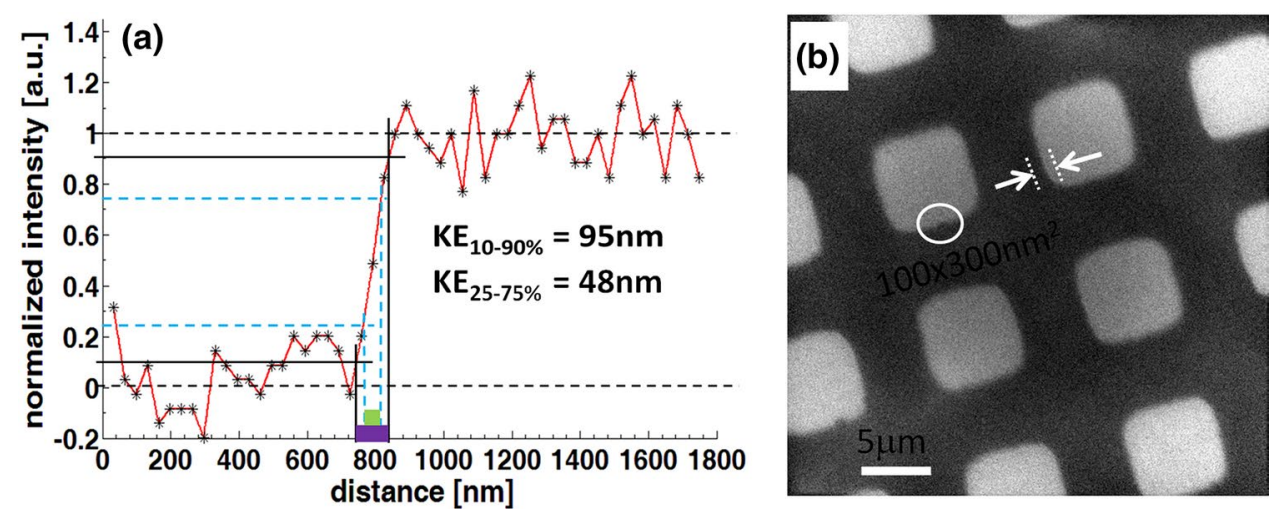

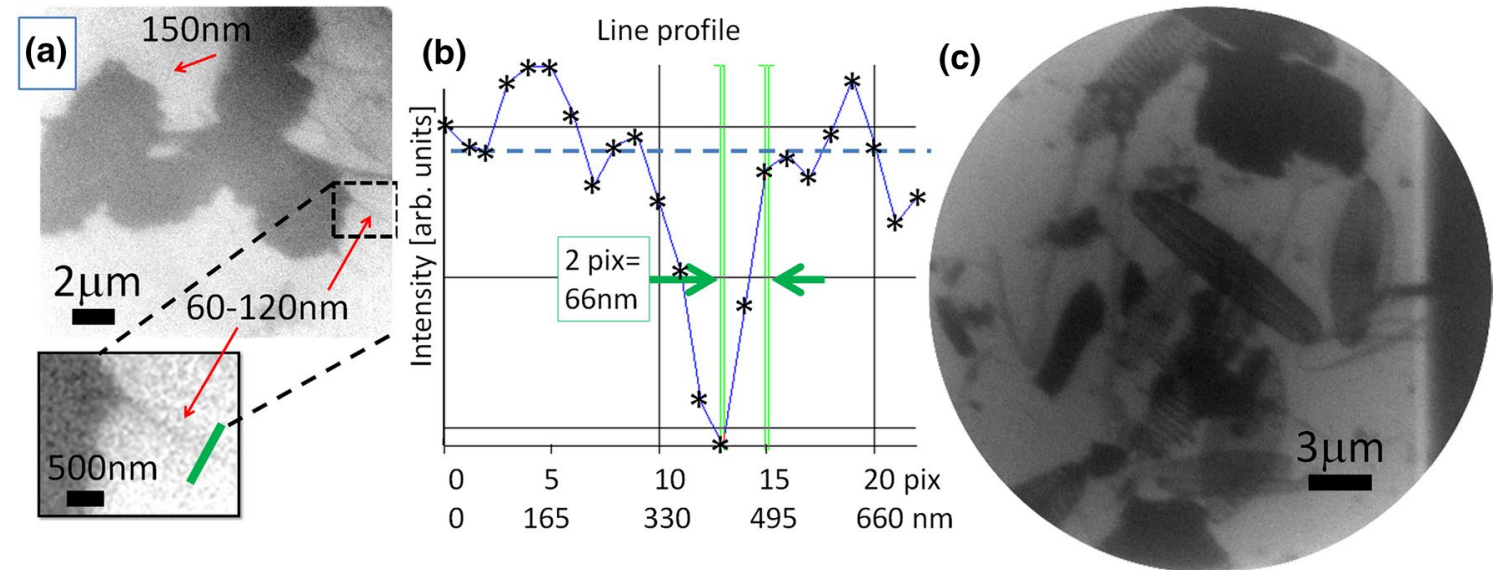

Fig. 4 The EUV image of CT 26 fibroblast cells (a), line profile through the smallest visible feature (b), indicating 0-95\% slope rise of $66 \mathrm{~nm}$. EUV image of diatoms (c) obtained with exposure of 200 EUV pulses $(20 \mathrm{~s})$, with visible features as small as $60 \mathrm{~nm}$

$95 \mathrm{~nm}, \sim 3$ pixels, fulfilling Nyquist-Shannon sampling criterion. The measured $25-75 \%$ intensity change was measured to be of the order of $\sim 48 \mathrm{~nm}$, almost an order of magnitude better than that for classical full-field visible light microscopes, limited by a wavelength to $\sim 300 \mathrm{~nm}$.

Diffraction-limited full-pitch spatial resolution of this system is equal to $1.22 \Delta r \sim 60 \mathrm{~nm}$. Our measurements, indicating $95 \mathrm{~nm}$, show that this resolution is $60 \%$ larger than the expected diffraction limit. This discrepancy is associated with the quality of the outer zones of the zone plate, fabricated with zone width-to-depth ratio equal to 4 in 200-nm-thick PMMA layer. Moreover, the EUV spectrum is not monochromatic, and other spectral components, shown in Fig. 1b, although weak comparing to the dominating $3 s-5 d \mathrm{Ar}^{7+}$ line, are affecting the quality of the EUV image as well.

This system was also successfully employed in imaging of real samples, such as CT 26 fibroblast cells from Mus musculus colon carcinoma (strain BALB/c), depicted in Fig. 4a, and diatoms (Fig. 4c) permitting to observe very small features, approaching the resolution limit of the microscope. The EUV images were acquired with 200 EUV pulses -20-s exposure time and detector temperature of $-20^{\circ} \mathrm{C}$. A sample was prepared by fixing CT 26 cells with $30 \%$ hexamethyldisilazane (HDMS) in absolute $\mathrm{EtOH}$ and placed on top of $30-\mathrm{nm} \mathrm{Si}_{3} \mathrm{~N}_{4}$ membrane. Small inset in the figure shows contrast-enhanced external feature of the CT 26 cell with the size varying from 60 to $120 \mathrm{~nm}$. Figure $4 \mathrm{~b}$ shows a line profile (trace) through the smallest visible feature, indicated with a green line, demonstrating 0-95\% slope rise of $66 \mathrm{~nm}$. Since this feature is not totally opaque and transmits some EUV radiation, it cannot be used as a proper test object for the resolution assessment based on the KE test; however, as can be noted, the slope rise from 0 to $95 \%$ indicates resolution of $\sim 70 \mathrm{~nm}$. Other examples of real nanostructures are the diatoms. The diatoms, depicted in Fig. 4b, were deposited from aqueous solution on top of $30-\mathrm{nm} \mathrm{Si}_{3} \mathrm{~N}_{4}$ membrane and dried in the nitrogen atmosphere for $5 \mathrm{~min}$. Both images show features approaching previously measured spatial resolution.

\section{Conclusions}

We demonstrate a novel, compact, desktop EUV transmission microscope, based on argon-helium double-stream gas-puff target EUV source and diffractive Fresnel optic. Such nanoimaging system, under incoherent illumination at $13.8 \mathrm{~nm}$ wavelength, acquires images of objects with a full-pitch spatial resolution better than $100 \mathrm{~nm}$, demonstrating superior spatial resolution to visible light microscopes, without the necessity to employ complicated and time-consuming serial acquisition optical schemes, such as STED.

The EUV microscope requires no specific sample preparation, such as conductive coating for SEM or staining the sample with fluorescence markers for achieving super-resolution in STED [29]. It is still a photon-based approach, which is important in certain types of applications requiring photon illumination such as imaging of thin layers of electron beam photoresists. The short attenuation length of EUV photons enhances optical contrast in extremely thin layers and features.

It is already well established and regarded that the water-window radiation is highly suitable for imaging of biological samples. It is true for imaging internals of the cells, due to relatively long attenuation lengths in water. We have shown that the EUV microscopy can also be used for imaging biological samples. It is more adequate, however, for imaging thin external cell features, such as morphology 
of the cellular membranes or other external features, such as flagella. In our opinion, such EUV microscope can be regarded as a complementary imaging tool to already existing, well-established ones. It can be employed, for example, in thin layer studies, providing additional information to optical and SEM microscopy. The compact systems, such as the one presented herein, are of major importance due to a possible widespread applicability, allowing potential rapid future developments in science and technology.

Acknowledgements We thank Šárka Vondrová, Jana Turňová and Prof. Miroslava Vrbová from Czech Technical University in Prague, Czech Republic, for preparing the cell samples investigated with the EUV full-field microscope and Dr. E. Viaggiu, University of Tor Vergata, Rome (Italy), AlgaRes srl, for the preparation of diatoms sample.

This work is supported by the National Centre for Research and Development, LIDER programme, Grant \#LIDER/004/410/L-4/12/ NCBR/2013, the National Science Centre, Opus programme, Grant agreement number UMO-2015/17/B/ST7/03718 and UMO2015/19/B/ST3/00435, the Education, Audiovisual and Culture Executive Agency (EACEA) Erasmus Mundus Joint Doctorate Programme Project No. 2012-0033 and from the European Union's Horizon 2020 research and innovation program, under Laserlab-Europe IV, Grant agreement No. 654148.

Open Access This article is distributed under the terms of the Creative Commons Attribution 4.0 International License (http://creativecommons.org/licenses/by/4.0/), which permits unrestricted use, distribution, and reproduction in any medium, provided you give appropriate credit to the original author(s) and the source, provide a link to the Creative Commons license, and indicate if changes were made.

\section{References}

1. ISO International Standard 21348

2. D. Attwood, Soft X-Rays and Extreme Ultraviolet Radiation (Cambridge University, Cambridge, 1999)

3. M.C. Marconi, P.W. Wachulak, Prog. Quantum Electron. 34(4), 173-190 (2010)

4. M.P. Benk, K. Goldberg, A. Wojdyla, C.N. Anderson, F. Salmassi, P. Naulleau, M. Kocsis, J. Vac. Sci. Technol. B 33(6), 06FE01 (2015)

5. W. Chao, P. Fischer, T. Tyliszczak, S. Rekawa, E. Anderson, P. Naulleau, Opt. Express 20(9), 9777-9783 (2012)

6. M. Toyoda, K. Yamasoe, A. Tokimasa, K. Uchida, T. Harada, T. Terasawa, T. Amano, T. Watanabe, M. Yanagihara, H. Kinoshita, Appl. Phys. Express 7(10), 102502 (2014)

7. M.P. Benk, A. Wojdyla, W. Chao, F. Salmassi, S. Oh, Y. Wang, R.H. Miyakawa, P. Naulleau, K.A. Goldberg, J. Micro/Nanolithogr. MEMS MOEMS 15(3), 033501 (2016)

8. Z.J. Qi, J.H. Rankin, M. Lawliss, K.D. Badger, Ch. Turley, J. Micro/Nanolithogr. MEMS MOEMS 15(2), 023502 (2016)

9. J. Kirz, Nat. Phys. 2, 799-800 (2006)
10. H.N. Chapman, A. Barty, M.J. Bogan, S. Boutet, M. Frank, S.P. Hau-Riege, S. Marchesini, B.W. Woods, S. Bajt, W.H. Benner, R.A. London, E. Plönjes, M. Kuhlmann, R. Treusch, S. Düsterer, T. Tschentscher, J.R. Schneider, E. Spiller, T. Möller, Ch. Bostedt, M. Hoener, D.A. Shapiro, K.O. Hodgson, D. van der Spoel, F. Burmeister, M. Bergh, C. Caleman, G. Huldt, M.M. Seibert, F.R.N.C. Maia, R.W. Lee, A. Szöke, N. Timneanu, J. Hajdu, Nat. Phys. 2, 839-843 (2006)

11. M. Wieland, Ch. Spielmann, U. Kleineberg, Th Westerwalbesloh, U. Heinzmann, T. Wilhein, Ultramicroscopy 102, 93-100 (2005)

12. J.J. Park, D.S. Kim, SCh. Jeon, J. Park, K.H. Lee, J. Lee, K.N. Kim, J.J. Yoo, ChH Nam, Opt. Lett. 34(3), 235-237 (2009)

13. M. Zurch, J. Rothhardt, S. Hadrich, S. Demmler, M. Krebs, J. Limpert, A. Tunnermann, A. Guggenmos, U. Kleineberg, C. Spielmann, Sci. Rep. 4, 7356 (2014)

14. P.W. Wachulak, C.A. Brewer, F. Brizuela, W. Chao, E. Anderson, R.A. Bartels, C.S. Menoni, J.J. Rocca, M.C. Marconi, J. Opt. Soc. Am. B 25, B20 (2008)

15. L. Juschkin, R. Freiberger, K. Bergmann, J. Phys. Conf. Ser. 186, 012030 (2009)

16. T. Ejima, F. Ishida, H. Murata, M. Toyoda, T. Harada, T. Tsuru, T. Hatano, M. Yanagihara, M. Yamamoto, H. Mizutani, Opt. Express 18(7), 7203-7209 (2010)

17. M. Müller, T. Mey, J. Niemeyer, K. Mann, Opt. Express 22(19), 23489 (2014)

18. M. Müller, T. Mey, J. Niemeyer, M. Lorenz, K. Mann, AIP Conf. Proc. 1764, 030003 (2016). doi:10.1063/1.4961137

19. H. Legall, G. Blobel, H. Stiel, W. Sandner, C. Seim, P. Takman, D.H. Martz, M. Selin, U. Vogt, H.M. Hertz, D. Esser, H. Sipma, J. Luttmann, M. Höfer, H.D. Hoffmann, S. Yulin, T. Feigl, S. Rehbein, P. Guttmann, G. Schneider, U. Wiesemann, M. Wirtz, W. Diete, Opt. Express 20(16), 18362-18369 (2012)

20. P.W. Wachulak, A. Bartnik, H. Fiedorowicz, T. Feigl, R. Jarocki, J. Kostecki, R. Rakowski, P. Rudawski, M. Sawicka, M. Szczurek, A. Szczurek, Z. Zawadzki, Appl. Phys. B 100(3), 461-469 (2010)

21. P.W. Wachulak, A. Bartnik, H. Fiedorowicz, Opt. Lett. 35(14), 2337-2339 (2010)

22. P.W. Wachulak, A. Torrisi, A. Bartnik, D. Adjei, J. Kostecki, Ł. Wegrzynski, R. Jarocki, M. Szczurek, H. Fiedorowicz, Appl. Phys. B 118(4), 573-578 (2015)

23. A. Bartnik, H. Fiedorowicz, T. Fok, R. Jarocki, J. Kostecki, A. Szczurek, M. Szczurek, P. Wachulak, Ł. Węgrzyński, Proc. SPIE 9441, 94410R (2014)

24. P.W. Wachulak, A. Bartnik, M. Skorupka, J. Kostecki, R. Jarocki, M. Szczurek, Ł. Węgrzyński, T. Fok, H. Fiedorowicz, Appl. Phys. B 111(2), 239-247 (2013)

25. H. Fiedorowicz, A. Bartnik, R. Jarocki, J. Kostecki, J. Krzywiński, J. Mikołajczyk, R. Rakowski, A. Szczurek, M. Szczurek, J. Alloys. Compd. 401, 99-103 (2005)

26. CXRO X-Ray Interactions With Matter website: http://henke.lbl. gov/optical_constants/

27. J.M. Heck, D.T. Attwood, W. Meyer-Ilse, E.H. Anderson, J. X-Ray Sci. Technol. 8, 95 (1998)

28. R.L. Kelly, J. Phys. Chem. Ref. Data. 16, Suppl 1 (1987)

29. J. Hanne, H.J. Falk, F. Görlitz, P. Hoyer, J. Engelhardt, S.J. Sahl, S.W. Hell, Nat. Commun. 6, 7127 (2015) 